Research Article

\title{
Prevalence and Associated Risk Factors of Intestinal Parasitic Infections among Pregnant Women Attending Antenatal Care in Yifag Health Center, Northwest Ethiopia
}

\author{
Destaw Damtie $\mathbb{D}^{1}$ and Minichil Liyih ${ }^{2}$ \\ ${ }^{1}$ Department of Biology, Bahir Dar University, Bahir Dar, Amhara Regional State, Ethiopia \\ ${ }^{2}$ Abune Gorgorios School, Bahir Dar City Administration, Bahir Dar, Amhara Regional State, Ethiopia \\ Correspondence should be addressed to Destaw Damtie; zegades96@gmail.com
}

Received 10 August 2021; Accepted 12 October 2021; Published 21 October 2021

Academic Editor: Louis Detolla

Copyright (c) 2021 Destaw Damtie and Minichil Liyih. This is an open access article distributed under the Creative Commons Attribution License, which permits unrestricted use, distribution, and reproduction in any medium, provided the original work is properly cited.

\begin{abstract}
Background. A cross-sectional study was conducted from November 2019 to March 2020 to determine the prevalence and associated risk factors of intestinal parasitic infections (IPIs) among pregnant women attending antenatal care (ANC) at Yifag Health Center. Methods. The data were collected by a questionnaire interview technique and collecting the stool samples from each pregnant woman. Wet-mount and formol-ether concentration techniques were applied to identify the IPIs. Data were analyzed using SPSS, version 25, and $P$-values $<0.05$ were considered statistically significant. Results. Out of the total 280 pregnant women who were selected using a simple random sampling technique, 277 participated in the questionnaire survey and gave stool samples (a response rate of 98.9\%). The prevalence of IPIs among pregnant women was 53.4\% (95\% CI: 47.37, 59.42). Taenia species (18.1\%) was the predominant parasite followed by Giardia lamblia (12.6\%), Entamoeba histolyticaldispar (9.4\%), hookworms (9\%), Ascaris lumbricoides (4\%), Schistosoma mansoni (3.2\%), Hymenolepis nana (0.7\%), Strongyloides stercoralis (0.4\%), and Enterobius vermicularis $(0.4 \%)$. Eating raw vegetables $(\mathrm{AOR}=2.721 ; 95 \% \mathrm{CI}: 1.266,5.849 ; P=0.010)$ and poor personal hygiene $(\mathrm{AOR}=4.015 ; 95 \% \mathrm{CI}: 1.456,11.07 ; P=0.007)$ were associated risk factors for $G$. lamblia, while eating raw meat $(\mathrm{AOR}=2.477$; 95\% CI: 1.252, 4.902; $P=0.009)$ for Taenia species infections. The prevalence of intestinal parasites was high and still a health burden to the pregnant women in the study area. We recommend avoiding eating raw meat, strengthening sanitation and hygiene programs, and routine deworming of pregnant mothers to reduce the burden of IPIs among pregnant women.
\end{abstract}

\section{Introduction}

Amebiasis, ascariasis, hookworm, and trichuriasis are among the top ten common intestinal parasitic infections (IPIs) [1]. IPIs caused by pathogenic helminth and protozoan species are endemic throughout the world, peculiarly in developing countries $[2,3]$. About one-fourth of the world's population lives with intestinal parasites [4].

IPIs during pregnancy, if left untreated, can cause adverse effects for the mothers, fetuses, and newborns. Globally, the three most prevalent helminth infections among pregnant women are hookworm (19\%), A. lumbricoides (17\%), and T. trichiura (11\%), and the three predominant protozoan infections are Blastocystis sp. (21\%), E. histolytica/dispar (9\%), and Giardia sp. (8\%) [5].

In Ethiopia, the overall prevalence of IPIs among pregnant women is unknown. But separate studies have been conducted in various localities. For example, the overall prevalence of IPIs among pregnant women attending prenatal care at Felege Hiwot Referral Hospital, Bahir Dar, was $31.5 \%$ [6]; among pregnant women attending ANC at public health facilities in the Lalo Kile district, Oromia, Western Ethiopia, was 43.8\% [7]; among pregnant women in West Gojjam Zone, Northwest Ethiopia, was 37.3\% [8], and among pregnant women in the Wondo Genet district, Southern Ethiopia, was 38.7\% [9]. 
The most predominant IPIs identified were G. lamblia (13.3\%) followed by E. histolytica/dispar (7.8\%), hookworms (5.5\%), A. lumbricoides (2.9 \%), S. mansoni (2.9\%), S. stercoralis (1.6\%), Taenia spp. (0.8\%), and H. nana $(0.3 \%)$ among pregnant women attending Felege Hiwot Referral Hospital [6]; hookworms (33.7\%) followed by A. lumbricoides (7.3\%) among pregnant women attending ANC at public health facilities in the Lalo Kile district, Oromia, Western Ethiopia [7]; hookworms (18.6\%) followed by E. histolytica/dispar (15.2\%) among pregnant women in West Gojjam Zone, Northwest Ethiopia [8]; and A. lumbricoides (24.9\%) followed by hookworms (11.2\%), G. lamblia (5.4\%), E. histolytica (3.4\%), T. trichiura (2.9\%), and S. mansoni (2.3\%) among pregnant women in the Wondo Genet district, Southern Ethiopia [9].

The distribution of IPIs depends on many factors such as low socioeconomic status, poor sanitation and personal hygiene, and lack of clean water [10]. The risk factors among pregnant women were being a farmer, walking barefooted, absence of proper handwashing after latrine [7], dwelling in a rural area, eating raw vegetables, improper use of toilets, poor environmental sanitation, the habit of soil eating, having irrigation practice, and lack of health education [8].

The aim of this study, therefore, was to determine the prevalence and associated risk factors of IPIs among pregnant women attending ANC at Yifag Health Center. The findings from this study would provide information about the status of IPIs and their associated risk factors among pregnant women in the Amhara region, serve as a springboard for Libokemkem District health officers and regional health officers, and will provide baseline information for further studies.

\section{Methods}

2.1. Study Area, Design, and Population. The location of Yifag Health Center is in Libokemkem District, South Gondar Zone, North West Ethiopia, at $12^{\circ} 5^{\prime} 0^{\prime \prime}$ latitude north and $37^{\circ} 44^{\prime} 0^{\prime \prime}$ longitude east. Its elevation ranges from 1829 to 1868 meters above sea level. It lies $77 \mathrm{~km}$ east of Bahir Dar, the capital city of the Amhara region, and $645 \mathrm{~km}$ northwest of Addis Ababa, the capital city of Ethiopia. It has a mean rainfall of $1330 \mathrm{ml}$ and a mean temperature ranging from 22 to $30^{\circ} \mathrm{C}$. There are five kebeles (lowest administrative units) whose residents are getting service from the health center. These kebeles harbor a total population of 198,374 (97,423 females, 100,951 males) [11]. Most of the residents of the study area are farmers who use mixed agriculture. The main cultivated crops are rice, maize, chickpea, oats (rye), and little irrigation practice of onion and garlic. Dug well and pipe waters are the sources of drinking water in the study area. But most people use river water for hygienic purposes. The sanitary facilities in the study area are open latrine systems. There are six health posts and one health center in the study area.

A health center-based cross-sectional study was conducted from 30 November 2019 to 07 March 2020. All the women visiting Yifag Health Center during the study period were the source population, while all the pregnant women were the study population.
2.2. Inclusion and Exclusion Criteria. The study included all pregnant women who were willing to give stool, sign informed consent, and did not take antiparasitic drugs for the last three weeks before screening.

2.3. Sample Size Determination and Sampling Technique. The sample size calculation used the single population proportion formula considering 95\% CI and $P=0.21$ [12] and adding $10 \%$ assumption of nonresponse rate as follows:

$$
n=\left(z_{\alpha / 2}\right)^{2} p \frac{(1-p)}{d^{2}}=255+10 \%(255)=280
$$

where $n$ is the sample size, $z_{\alpha / 2}=1.96$ for the standard scale of $95 \%$ level of confidence, and $d$ is the marginal error, which is 0.05 .

2.4. Collection of Stool Samples. The respondents were given clear instructions and provided with clean labeled collection cups along with applicator sticks. Each mother delivered about four grams of a fresh stool sample. The data collector registered the date of sampling, the name of each participant, and the age of each participant during data collection. The WHO guideline [13] was employed to process and examine a portion of each of the stool samples using direct wet-mount and formol-ether concentration techniques in the $\mathrm{YHC}$ laboratory class for parasitological examination.

2.5. Questionnaire Survey. The researchers developed a structured questionnaire first in the English language. The questionnaire incorporated issues about the sociodemography, water source, personal hygiene, latrine availability, residence, attitudes on IPIs, basic knowledge on common signs and symptoms of IPIs, pregnancy month, and interpregnancy of the respondents. Then, it was translated into the local language (Amharic) for interview. Before the interview, the questionnaires were pretested among thirty mothers. Then, we made the necessary adjustments based on the feedback. The data collectors interviewed the respondents during stool collection. We translated the responses back into English for data analysis.

2.6. Direct Microscopy (Wet Mount) Procedure. In the wet mount, a fresh stool sample of each participant (about $2 \mathrm{mg}$ ) was placed on a glass slide with a wooden applicator, emulsified with a drop of physiological saline $(0.85 \%)$ for diarrheic and semisolid samples. For formed stools, iodine was used. Then, covered with cover slide and examined for the presence of motile intestinal parasites and trophozoites under a microscope using first X10 objectives and then X40 objectives [14].

2.7. Formol-Ether Concentration Technique. A portion of each stool sample was used for the detection of parasitic ova and protozoan cysts using the formol-ether concentration technique. About 2 grams of each stool sample was first emulsified with three to four $\mathrm{ml}$ of $10 \%$ formol saline. This 
was mixed thoroughly and passed through the gauze [14]. Three to four $\mathrm{ml}$ of diethyl ether was added and mixed by inverting and intermittent shaking for one minute and then centrifuged at 3,000 rpm for five minutes. After centrifugation, the supernatant (layers of ether, debris, and formol saline) was discarded by a pipette and the sediment (containing the parasites at the bottom of the test tube) was resuspended in formol saline. The sediment was examined microscopically under $10 \mathrm{X}$ by $10 \mathrm{X}$ and $10 \mathrm{X}$ by $40 \mathrm{X}$ magnifications for the presence of any parasitic organisms [14]. To maintain the reliability of the study findings, the specimen was reexamined at the end by an experienced laboratory technologist who was blind for the first examination result.

2.8. Variables. The prevalence of the parasitic infections was the dependant variable, while associated risk factors, sociodemographic factors (age, residence, educational level, family size, and religion), socioeconomic factors (occupation, access to clean water, access to the toilet, knowledge about IPIs), environmental factors (source of water), and behavioral factors (hand wash before food and after toilet, eating raw food, personal hygiene, shoe-wearing habit, waste discharge habit, fingernail status) were independent variables.

2.9. Data Analysis. Statistical Package for Social Sciences (SPSS) version 25 was used to analyze the collected data. Descriptive statistics such as frequency, percentage, mean, and range were determined for each intestinal parasite. Binary logistic regression was used to measure the strengths of association between the prevalence of infection and the risk factors using the odds ratio. In the modeling process, a univariate analysis was first carried out with less than a 0.25 level of significance to select the candidate variables for multivariate analysis. The variables, significant at a $P$-value of $<0.25$ in the univariate analysis, were then included in the multivariate analysis [15]. Values were considered significant at $P<0.05$.

2.10. Ethical Consideration. Ethical clearance was obtained from the Ethical Review Committee of the Science College, Bahir Dar University, before data collection (S1_File). A letter describing the objective of the study was written to the Libokemkem Health Office and Yifag Health Center. The researcher obtained consent from the study participants after explaining the purposes and the procedures of the study. The laboratory test and the questionnaires were conducted with strict privacy and confidentiality. The pregnant women whose test results are positive were given standard drugs free of charge.

\section{Results}

3.1. Sociodemographic Characteristics of the Study Participants. A total of 277 (99\%) pregnant women gave stool for intestinal parasitic examination and filled the questionnaires. The mean age of the study subjects was
26.6 years. Two hundred four $(73.6 \%)$ were farmers, 22 (7.9\%) housewives, 9 (3.3\%) government employees, and 42 (15.2\%) merchants. Two hundred thirteen (76.9\%) and 64 (23.1\%) of the participants had family sizes of $\leq 5$ and $>5$, respectively. The educational status of the participants was illiterate (63.2\%), primary school (20.9\%), secondary school (10.5\%), and diploma and above (5.4\%) (Table 1).

3.2. Hygienic Characteristics of the Respondents. The proportions of the respondents for hygienic characteristics were access to protected water (93.1\%), access to toilet (6.9\%), personal hygiene (good/poor: 33.2\%/66.8\%), awareness about parasitic infections (good/poor: 19.5\%/80.5\%), handwashing habit (before feeding $=99.6 \%$, after defecation $=58.8 \%$, not at all $=41.2 \%$ ), habit of wearing shoes (regularly $=54.2 \%$, not at all $=45.8 \%$ ), waste disposal mechanism (burry/burn $=9.0 \%$, open field disposal $=91.0 \%$ ), fingernail status (trimmed $=58.5 \%$, untrimmed $=41.5 \%$ ), and raw meat $/$ vegetable feeding habit $(40.1 \% / 45.8 \%)$ (Table 2$)$.

3.3. Prevalence of IPIs in the Study Population. Two species of protozoa and seven species of intestinal helminths were identified from the stool samples of the study participants. The overall prevalence of IPIs was $53.4 \%$. Taenia species $(18.1 \%)$ and G. lamblia (12.6\%) were the two top and E. vermicularis $(0.4 \%)$ and S. stercoralis $(0.4 \%)$ were the least prevalent IPIs. The prevalence of protozoa and helminths was $22.0 \%$ and $35.7 \%$, respectively. The rates of the single, double, and triple infections were $49.5 \%, 3.6 \%$, and $0.4 \%$, respectively (Table 3 ). Most of the double infections occurred between the Tania species and others, and triple infections occurred among E. histolytica/dispar, G. lamblia, and hookworms.

3.4. Factors Associated with IPIs among Pregnant Women Visiting YHC for ANC. In both the univariate and multivariate logistic regressions, no specific risk factors were observed for E. histolytica/dispar, hookworms, A. lumbricoides, and $S$. mansoni $(P>0.05)$. Risk factors were identified to G. lamblia and Taenia species $(P<0.05)$ (Table 4$)$. The multivariate logistic regression showed that mothers who had the habit of eating raw vegetables were 2.721 times more infected by G. lamblia (AOR $=2.721 ; 95 \%$ CI: 1.266, 5.849; $P=0.010)$ than their counterparts, and mothers who had poor personal hygiene were four times more likely infected (AOR $=4.015 ; 95 \%$ CI: $1.456,11.07 ; P=0.007)$ by G. lamblia than those with good personal hygiene (Table 4). Furthermore, mothers who had the habit of eating raw meat were 2.5 times $(\mathrm{AOR}=2.477 ; 95 \% \mathrm{CI}: 1.252,4.902 ; P=0.009)$ more likely to be infected by the Taenia species than their contemporaries.

\section{Discussion}

The overall prevalence of IPIs in this study was 53.4\% (95\% CI: $47.4,59.4)$. It was comparable with previous studies conducted in North Ethiopia (51.5\%) [16] and Nigeria 
TABLE 1: Sociodemographic profile of pregnant women at Yifag Health Center, Northwest Ethiopia $(N=277)$, from November 2019 to March 2020.

\begin{tabular}{|c|c|c|c|}
\hline Variable & Categories & Frequency & Percent \\
\hline \multirow{2}{*}{ Age group } & $18-30$ & 224 & 80.9 \\
\hline & $31-45$ & 53 & 19.1 \\
\hline \multirow{5}{*}{ Residence } & Around Yifag & 43 & 15.5 \\
\hline & Bura & 92 & 33.2 \\
\hline & Ginaza & 19 & 6.9 \\
\hline & Shina & 28 & 10.1 \\
\hline & Yifag & 95 & 34.3 \\
\hline \multirow{2}{*}{ Religion } & Muslim & 17 & 6.1 \\
\hline & Orthodox & 260 & 93.9 \\
\hline \multirow{4}{*}{ Occupation } & Agriculture & 204 & 73.6 \\
\hline & Government employed & 9 & 3.2 \\
\hline & Housewives & 22 & 7.9 \\
\hline & Merchant & 42 & 15.2 \\
\hline \multirow{4}{*}{ Educational level } & Diploma and above & 15 & 5.4 \\
\hline & Illiterate & 175 & 63.2 \\
\hline & Primary & 58 & 20.9 \\
\hline & Secondary & 29 & 10.5 \\
\hline \multirow{2}{*}{ Family no. } & $\leq 5$ & 213 & 76.9 \\
\hline & $>5$ & 64 & 23.1 \\
\hline
\end{tabular}

(56.8\%) [17]. But it was higher than the findings from the Gilgel Gibe Dam area, Southwest Ethiopia (11.6\%) [18]; Lalo Kile district, Western Ethiopia (43.8\%) [7]; Dembiya, Northwest Ethiopia (25.8\%) [19]; Colombia (41\%) [20]; Nepal (35\%) [2]; and Kenya (13.8\%) [21] and lower than the studies conducted in Mecha District, Northwest Ethiopia (70.6\%) [22]; Papua New Guinea (81.0\%) [23]; and Venezuela $(73.9 \%)[23,24]$. These variations might be due to the differences in the sociodemographic factors, lack of awareness on the prevention of parasitic infections, personal hygiene, eating uncooked food, not trimming fingernails, poor waste disposal, bare footedness, lack of clean water, and environmental factors.

The most prevalent IPI in this study was the Taenia species (18.1\%). A lower prevalence of Taenia species was reported from Arba Minch Town, Ethiopia (0.6\%) [25]; Bahir Dar, Northwest Ethiopia (0.8\%) [6]; East Wollega, Ethiopia (1.3\%) [26]; and Iran (0.014\%) [27]. This difference may be due to the differences in the habit of eating uncooked meat, altitude, open defecation, and poor awareness in the prevention of this parasite [28].

G. lamblia was the second most prevalent parasite $(12.6 \%)$ among the study participants. This finding was in line with previous studies conducted in Southern Ethiopia (12.6\%) [29]; Bahir Dar, Northwest Ethiopia (13.3\%) [6]; and Venezuela (14.1\%) [24]. It was lower than the prevalence in studies conducted in Northwest Ethiopia (19.2\%) [30] and Papua New Guinea (39\%) [23]. But it was higher than studies conducted in Wolayita Sodo Town, Southern Ethiopia (3.6\%) [31]; Lalo Kile district, Western Ethiopia (0.9\%) [7]; and Wondo Genet district, Southern Ethiopia (5.4\%) [9]. Accessibility of safe water, open field defecation, and variations in hand wash implementation might bring such differences [32].
E. histolytica/dispar infection among the study participants was $9.4 \%$. It was consistent with studies conducted in the Gamo area, Southern Ethiopia (11.4\%) [33]; Bahir Dar, Northwest Ethiopia (7.8\%) [6]; and Venezuela (12.0) [24]. But it was lower than the reports from Nigeria (18.9\% [17], 2010) and Papua New Guinea (43\%) [23] and higher than the findings from Southwest Ethiopia (5.5\%) [34]; Wondo Genet district, Southern Ethiopia (3.4\%) [9]; and Tanzania (0.7\%) [35]. Accessibility of safe water, open field defecation, variations in handwashing habits before food and after toilet, eating raw vegetables, contact with night soil, sanitation problems, and environmental and climate factors might bring such differences [36].

The level of hookworm in the present study was $9.0 \%$. This finding was in agreement with the reports from Southern Ethiopia (9.9\%) [29]; Wondo Genet district, Southern Ethiopia (11.2\%) [9]; and Venezuela (8.1\%) [24]. However, this prevalence was lower than that of the study conducted in Northwest Ethiopia (20\%) [12]; the Gilgel Gibe Dam area, Southwest Ethiopia (29.4\%) [18]; Lalo Kile district, Western Ethiopia (33.7\%) [7]; the Maytsebri primary hospital, North Ethiopia (39.96\%) [16]; Uganda (40.5\%) [37]; and Nigeria (44.4\%) [38]. But it was higher than the reports from Kenya (3.92\%) [21] and Bahir Dar, Northwest Ethiopia (5.5\%) [6]. This difference might be due to the differences in geography, shoe-wearing habits, level of income, and agricultural practices [7].

The prevalence of $A$. Lumbricoides in the present study was $4 \%$. It was comparable with the results of the reports from Wolayita Sodo Town, Southern Ethiopia (5.5\%) [31]; Bahir Dar, Northwest Ethiopia (2.9\%) [6]; and Kenya (6.5\%) [21]. But it was higher than the findings in Arba Minch Town, Ethiopia (0.3\%) [25] and in Ghana (0.9\%) [39] and lower than results from Lalo Kile district, Western Ethiopia (7.3\%) [7]; the Gilgel Gibe Dam area, Southwest Ethiopia (15\%) [18]; the Maytsebri primary hospital, North Ethiopia (12.7\%) [16]; Wondo Genet district, Southern Ethiopia (24.9\%) [9]; Mecha District, Northwest Ethiopia (32.2\%) [22]; Nigeria (52.2\%) [38]; and Venezuela (57.0) [24]. The observed difference might be due to environmental conditions and environmental sanitation problems, differences in eating raw vegetables, lack of handwashing, agricultural practices, waste disposal habit, lack of clean waters, and open defecations $[22,40]$.

The prevalence of S. mansoni was 3.2\%. It was comparable with studies conducted in Bahir Dar, Northwest Ethiopia (2.9\%) [6], and Jimma, Southwest Ethiopia (3.6\%) [34]. However, it was higher than the finding from Northwest Ethiopia (2.2\%) [12]; Wondo Genet district, Southern Ethiopia (2.3\%) [9]; and Wolayita Sodo Town, Southern Ethiopia (0.6\%) [31]. But it was lower than that of the studies conducted in Mecha District, Northwest Ethiopia (17.4\%) [22], Waja-Timuga, District of Alamata, Northern Ethiopia (73.9\%) [41]; Cameroon (28.01\%) [42]; and Uganda (36.4\%) [37]. The variations in S. mansoni infection could be due to the differences in the geographical areas, environmental pollution with urine and feces, the habit of crossing and bathing in river waters, eating uncooked vegetables and foods, and cercariae-infested water sources [41]. 
TABle 2: Practice of pregnant women related to personal and environmental hygiene taking ANC at Yifag Health Center, Northwest Ethiopia $(N=277)$, November 2019 to March 2020.

\begin{tabular}{|c|c|c|c|}
\hline Variable & Categories & Frequency & Percent \\
\hline \multirow{2}{*}{ Waste disposal } & Burring or burning & 25 & 9.0 \\
\hline & Open field & 252 & 91.0 \\
\hline \multirow{2}{*}{ Have toilet } & No & 206 & 74.4 \\
\hline & Yes & 71 & 25.6 \\
\hline \multirow{3}{*}{ Type of toilet } & Private & 31 & 11.2 \\
\hline & Open field & 209 & 75.5 \\
\hline & Public & 37 & 13.4 \\
\hline \multirow{2}{*}{ Source of water } & Pipe & 258 & 93.1 \\
\hline & Unprotected & 19 & 6.9 \\
\hline \multirow{2}{*}{ Handwashing before feeding } & No & 1 & 0.4 \\
\hline & Yes & 276 & 99.6 \\
\hline \multirow{2}{*}{ Handwashing after toilet } & No & 163 & 58.8 \\
\hline & Yes & 114 & 41.2 \\
\hline \multirow{2}{*}{ Fingernail status } & Trimmed & 162 & 58.5 \\
\hline & Untrimmed & 115 & 41.5 \\
\hline \multirow{2}{*}{ Eating raw meat } & No & 166 & 59.9 \\
\hline & Yes & 111 & 40.1 \\
\hline \multirow{2}{*}{ Eating raw vegetables } & No & 150 & 54.2 \\
\hline & Yes & 127 & 45.8 \\
\hline \multirow{2}{*}{ Personal hygiene } & Good & 92 & 33.2 \\
\hline & Poor & 185 & 66.8 \\
\hline \multirow{2}{*}{ Shoe wearing } & No & 127 & 45.8 \\
\hline & Yes & 150 & 54.2 \\
\hline \multirow{2}{*}{ Transmission and prevention knowledge of IPI } & No & 223 & 80.5 \\
\hline & Yes & 54 & 19.5 \\
\hline
\end{tabular}

TABLE 3: Prevalence of IPIs among pregnant women $(N=277)$ attending ANC in Yifag Health Center, Northwest Ethiopia, November 2019 to March 2020.

\begin{tabular}{lcc}
\hline Parasite & $\begin{array}{c}\text { Number women } \\
\text { infected }\end{array}$ & Percent \\
\hline Protozoa & 26 & \\
E. histolytica/dispar & 35 & 9.4 \\
G. lamblia & 61 & 12.6 \\
Total & & 22 \\
\hline Helminths & 11 & \\
A. lumbricoides & 25 & 4.0 \\
Hookworm & 2 & 9.0 \\
H. nana & 9 & 0.7 \\
S. mansoni & 50 & 3.2 \\
Taenia spp. & 1 & 18.1 \\
E. vermicularis & 1 & 0.4 \\
S. stercoralis & 99 & 0.4 \\
Total & & 35.7 \\
\hline Single and multiple infections & 137 & \\
Single infections & 10 & 49.5 \\
Double infections & 1 & 3.6 \\
Triple infections & 148 & 0.4 \\
\hline The overall prevalence of IPIs & & 53.4 \\
\hline
\end{tabular}

The prevalence of $H$. nana in this study was $0.7 \%$. This prevalence agreed with the findings from Gondar Town, Northwest Ethiopia (0.5\%) [43], and Bahir Dar, Northwest Ethiopia $(0.3 \%)$ [6]. However, the findings of studies reported from the Gilgel Gibe Dam area, Southwest Ethiopia (1.6\%) [18]; East Wollega, Oromia, Ethiopia (1.6\%) [26]; and Baghdad/Iraq (6.67\%) [44] were higher than the current finding. These variations might be due to the variations in the maintenance of good personal hygiene and sanitary improvements, consumption of contaminated food and water, and rodent control [45].

The prevalence of $S$. stercoralis in the current finding was $0.4 \%$, which was in line with the study conducted in East Wollega, Ethiopia (0.3\%) [26], and Lalo Kile district, Western Ethiopia (0.3\%) [7]. But it showed a lower prevalence of $S$. stercoralis than that found in studies conducted in Bahir Dar, Northwest Ethiopia (1.6\%) [6]; Mecha District, Northwest Ethiopia (6.4\%) [22]; Nigeria (1.3\%) [38]; Papua New Guinea (3\%) [23]; and Venezuela (3.3\%) [24]. These variations may be accounted for by the differences in contamination of the soil with feces, walking barefoot, open latrine system, and environmental sanitation [46].

In this study, the prevalence of $E$. vermicularis was $0.4 \%$. It was consistent with the study conducted in the Gilgel Gibe Dam area, Southwest Ethiopia (0.3\%) [18]. However, it was lower than that found in other studies reported from Nigeria (3.5\%) [47], Kenya (4.8\%) [21], and Venezuela (6.3\%) [24] and far lower than Iraq (32.9\%) [48]. These variations in the prevalence of $E$. vermicularis among studies could be due to the differences in environmental sanitation, parasitological methods used during the study, and maintenance of 
TABLE 4: Univariate and multivariate logistic regression analysis of potential risk factors associated with IPIs among pregnant women in Yifag Health Center, Northwest Ethiopia, November 2019 to March 2020.

\begin{tabular}{|c|c|c|c|c|c|c|c|c|}
\hline \multirow[t]{2}{*}{ Risk factors } & \multirow[t]{2}{*}{ Categories } & \multirow[t]{2}{*}{ Total } & \multirow{2}{*}{$\begin{array}{c}\text { Negative no. } \\
(\%)\end{array}$} & \multirow{2}{*}{$\begin{array}{c}\text { Positive no. } \\
(\%)\end{array}$} & \multicolumn{2}{|c|}{$\begin{array}{l}\text { Univariate logistic } \\
\text { regression }\end{array}$} & \multicolumn{2}{|c|}{$\begin{array}{c}\text { Multivariate logistic } \\
\text { regression }\end{array}$} \\
\hline & & & & & COR $(95 \%$ CI $)$ & $P$-value & $\operatorname{AOR}(95 \% \mathrm{CI})$ & $P$-value \\
\hline \multicolumn{9}{|l|}{ E. histolytica/dispar } \\
\hline \multirow{3}{*}{ Type of toilet } & Private & 31 & $25(80.6)$ & $6(19.4)$ & 1 & \multirow{3}{*}{0.149} & 1 & \multirow{3}{*}{0.149} \\
\hline & Open field & 209 & $192(91.9)$ & $17(8.1)$ & $0.369(0.133,1.023)$ & & $\begin{array}{c}0.369(0.133 \\
1.023)\end{array}$ & \\
\hline & Public & 37 & $34(91.9)$ & $3(8.1)$ & $0.368(0.084,1.613)$ & & $\begin{array}{c}0.368(0.084 \\
1.613)\end{array}$ & \\
\hline \multicolumn{9}{|l|}{ G. lamblia } \\
\hline \multirow[b]{2}{*}{ Fingernail status } & Trimmed & 162 & $146(90.1)$ & $16(9.9)$ & 1 & \multirow[b]{2}{*}{0.104} & - & \multirow[b]{2}{*}{0.111} \\
\hline & Untrimmed & 115 & $96(83.5)$ & $19(16.5)$ & $1.806(0.885,3.685)$ & & $\begin{array}{c}1.831(0.870 \\
3.853)\end{array}$ & \\
\hline \multirow{2}{*}{$\begin{array}{l}\text { Eating raw } \\
\text { vegetables }\end{array}$} & No & 150 & $136(90.7)$ & $14(9.3)$ & 1 & \multirow[b]{2}{*}{0.076} & 1 & \multirow[b]{2}{*}{$0.010^{*}$} \\
\hline & Yes & 127 & $106(83.5)$ & $21(16.5)$ & $1.925(0.935,3.963)$ & & $\begin{array}{l}2.721(1.266 \\
5.849)\end{array}$ & \\
\hline \multirow[b]{2}{*}{ Personal hygiene } & Good & 92 & $87(94.6)$ & $5(5.4)$ & 1 & \multirow[b]{2}{*}{$0.015^{*}$} & 1 & \multirow[b]{2}{*}{$0.007^{*}$} \\
\hline & Poor & 185 & $155(83.8)$ & $30(16.2)$ & $3.368(1.261,8.996)$ & & $\begin{array}{c}4.015(1.456 \\
11.07)\end{array}$ & \\
\hline \multicolumn{9}{|l|}{ A. lumbricoides } \\
\hline \multirow{2}{*}{$\begin{array}{l}\text { Hand wash after } \\
\text { toilet }\end{array}$} & No & 163 & $159(97.5)$ & $4(2.5)$ & $0.385(0.110,1.346)$ & \multirow[t]{2}{*}{0.135} & $\begin{array}{l}0.827(0.186 \\
3.676)\end{array}$ & \multirow[t]{2}{*}{0.803} \\
\hline & Yes & 114 & $107(93.9)$ & $7(6.1)$ & 1 & & 1 & \\
\hline & $\begin{array}{l}\text { Burial/ } \\
\text { burning }\end{array}$ & 25 & $22(88.0)$ & $3(12.0)$ & 1 & & 1 & 0.372 \\
\hline Waste disposal & Open field & 252 & $244(96.8)$ & $8(3.2)$ & $0.240(0.059,0.972)$ & $0.046^{\prime \prime}$ & $\begin{array}{l}0.487(0.100 \\
2.362)\end{array}$ & $0.3 / 2$ \\
\hline & Trimmed & 162 & $158(97.5)$ & $4(2.5)$ & 1 & & 1 & \\
\hline Fingernail status & Untrimmed & 115 & $108(93.9)$ & $7(6.1)$ & $2.560(0.732,8.959)$ & 0.141 & $\begin{array}{l}2.618(0.729 \\
9.409)\end{array}$ & 0.140 \\
\hline Knowledge about & No & 223 & $218(97.8)$ & $5(2.2)$ & $0.183(0.054,0.626)$ & $0.007^{*}$ & $\begin{array}{c}0.240(0.057 \\
1.005)\end{array}$ & 0.051 \\
\hline & Yes & 54 & $48(88.9)$ & $6(11.1)$ & 1 & & 1 & \\
\hline Hookworm & & & & & & & & \\
\hline & $18-30$ & 224 & $206(92)$ & $18(8)$ & 1 & & 1 & \\
\hline Age group & $31-45$ & 53 & $46(86.8)$ & $7(13.2)$ & $1.742(0.687,4.413)$ & 0.242 & $\begin{array}{c}1.520(0.593 \\
3.896)\end{array}$ & 0.383 \\
\hline & No & 166 & $154(92.8)$ & $12(7.2)$ & 1 & & 1 & \\
\hline Eating raw meat & Yes & 111 & $98(88.3)$ & $13(11.7)$ & $1.702(0.746,3.883)$ & 0.206 & $\begin{array}{c}1.713(0.746 \\
3.932)\end{array}$ & 0.204 \\
\hline Knowledge about & No & 223 & $200(89.7)$ & $23(10.3)$ & $\begin{array}{c}2.990(0.683 \\
13.092)\end{array}$ & 0.146 & $\begin{array}{c}2.871(0.648 \\
12.727)\end{array}$ & 0.165 \\
\hline IPIs & Yes & 54 & $52(96.3)$ & $2(3.7)$ & 1 & & 1 & \\
\hline S. mansoni & & & & & & & & \\
\hline & Agriculture & 204 & $200(98.0)$ & $4(2.0)$ & $0.400(0.071,2.259)$ & & - & \\
\hline Occupation & Housewives & 22 & $19(86.4)$ & $3(13.6)$ & $\begin{array}{c}3.158(0.486 \\
20.503)\end{array}$ & 0.083 & - & 0.518 \\
\hline & Merchant & 42 & $40(95.2)$ & $2(4.8)$ & - & & - & \\
\hline & Government & 9 & $9(100.0)$ & $0(0.0)$ & 1 & & 1 & \\
\hline Hand wash after & No & 163 & $160(98.2)$ & $3(1.8)$ & $0.338(0.083,1.379)$ & 0.130 & $\begin{array}{c}0.919(0.132 \\
6.389)\end{array}$ & 0.932 \\
\hline toilet & Yes & 114 & $108(94.7)$ & $6(5.3)$ & 1 & & 1 & \\
\hline Shoe wearing & No & 127 & $125(98.4)$ & $2(1.6)$ & $0.327(0.067,1.602)$ & 0.168 & $\begin{array}{c}0.709(0.104 \\
4.841)\end{array}$ & 0.725 \\
\hline & Yes & 150 & $143(95.3)$ & $7(4.7)$ & 1 & & 1 & \\
\hline Toilet & $\begin{array}{l}\text { Absent } \\
\text { Present }\end{array}$ & 206 & $201(97.6)$ & $5(2.4)$ & $0.417(0.109,1.597)$ & 0.202 & - & 0.999 \\
\hline
\end{tabular}


TABLE 4: Continued.

\begin{tabular}{|c|c|c|c|c|c|c|c|c|}
\hline \multirow[t]{2}{*}{ Risk factors } & \multirow[t]{2}{*}{ Categories } & \multirow[t]{2}{*}{ Total } & \multirow{2}{*}{$\begin{array}{c}\text { Negative no. } \\
(\%)\end{array}$} & \multirow{2}{*}{$\begin{array}{l}\text { Positive no. } \\
(\%)\end{array}$} & \multicolumn{2}{|c|}{$\begin{array}{l}\text { Univariate logistic } \\
\text { regression }\end{array}$} & \multicolumn{2}{|c|}{$\begin{array}{l}\text { Multivariate logistic } \\
\text { regression }\end{array}$} \\
\hline & & & & & COR $(95 \% \mathrm{CI})$ & $P$-value & AOR $(95 \% \mathrm{CI})$ & $P$-value \\
\hline \multirow{3}{*}{ Type of toilet } & Private & 31 & $30(96.8)$ & $1(3.2)$ & 1 & \multirow{3}{*}{0.233} & - & \multirow{3}{*}{0.745} \\
\hline & Open & 209 & $204(97.6)$ & $5(2.4)$ & $0.735(0.083,6.511)$ & & - & \\
\hline & Public & 37 & $34(91.9)$ & $3(8.1)$ & $\begin{array}{c}2.647 \\
(0.261,26.823)\end{array}$ & & $\begin{array}{l}2.614(0.225 \\
30.400)\end{array}$ & \\
\hline \multirow[b]{2}{*}{ Fingernail status } & Trimmed & 162 & $155(95.7)$ & $7(4.3)$ & 1 & \multirow[b]{2}{*}{0.248} & 1 & \multirow[b]{2}{*}{0.468} \\
\hline & Untrimmed & 115 & $113(98.3)$ & $2(1.7)$ & $0.392(0.080,1.922)$ & & $\begin{array}{c}0.537(0.100 \\
2.879)\end{array}$ & \\
\hline \multirow[b]{2}{*}{ Personal hygiene } & Good & 92 & $86(93.5)$ & $6(6.5)$ & 1 & \multirow[b]{2}{*}{$0.045^{*}$} & 1 & \multirow[b]{2}{*}{0.231} \\
\hline & Poor & 185 & $182(98.4)$ & $3(1.6)$ & $0.24(0.058,0.967)$ & & $\begin{array}{c}0.341(0.059 \\
1.98)\end{array}$ & \\
\hline \multicolumn{9}{|l|}{ Taenia species } \\
\hline \multirow{6}{*}{ Residence } & Around Yifag & 43 & $32(74.4)$ & $11(25.6)$ & $3.285(1.245,8.664)$ & \multirow{5}{*}{$0.033^{*}$} & $\begin{array}{c}2.051(0.485 \\
8.668)\end{array}$ & \multirow{6}{*}{0.510} \\
\hline & Bura & 92 & $70(76.1)$ & $22(23.9)$ & $3.003(1.300,6.937)$ & & $\begin{array}{c}1.616(0.410 \\
6.366)\end{array}$ & \\
\hline & Ginaza & 19 & $18(94.7)$ & $1(5.3)$ & $0.531(0.063,4.456)$ & & $\begin{array}{l}0.332(0.030 \\
3.710)\end{array}$ & \\
\hline & Shina & 28 & $21(75.0)$ & $7(25.0)$ & $3.185(1.064,9.539)$ & & $\begin{array}{c}1.691(0.344 \\
8.309)\end{array}$ & \\
\hline & Yifag & 95 & $86(90.5)$ & $9(9.5)$ & 1 & & 1 & \\
\hline & Muslim & 17 & $16(94.1)$ & $1(5.9)$ & 1 & \multirow[b]{2}{*}{0.208} & 1 & \\
\hline \multirow[t]{2}{*}{ Religion } & Orthodox & 260 & $211(81.2)$ & $49(18.8)$ & $\begin{array}{c}3.716(0.481 \\
28.692)\end{array}$ & & $\begin{array}{c}0.616(0.044 \\
8.542)\end{array}$ & 0.718 \\
\hline & Agriculture & 204 & $161(78.9)$ & $43(21.1)$ & $0.534(0.128,2.224$ & & $\begin{array}{c}0.108(0.013 \\
0.904)\end{array}$ & \multirow{4}{*}{0.063} \\
\hline \multirow[t]{3}{*}{ Occupation } & Housewives & 9 & $6(66.7)$ & $3(33.3)$ & $0.200(0.027,1.490)$ & \multirow[t]{2}{*}{0.057} & $\begin{array}{c}0.117(0.013 \\
1.045)\end{array}$ & \\
\hline & Merchant & 22 & $20(90.9)$ & $2(9.1)$ & $0.100(0.014,0.727)$ & & $\begin{array}{c}0.054(0.006 \\
0.489)\end{array}$ & \\
\hline & Government & 42 & $40(95.2)$ & $2(4.8)$ & 1 & & 1 & \\
\hline \multirow{2}{*}{$\begin{array}{l}\text { Hand wash after } \\
\text { toilet }\end{array}$} & No & 163 & $127(77.9)$ & $36(22.1)$ & $2.025(1.035,3.959)$ & $0.039^{*}$ & $\begin{array}{c}1.442(0.613 \\
3.395)\end{array}$ & 0.402 \\
\hline & Yes & 114 & $100(87.7)$ & $14(12.3)$ & 1 & & 1 & \\
\hline Shoes wearing & No & 127 & $96(75.6)$ & $31(24.4)$ & $2.226(1.187,4.176)$ & $0.013^{*}$ & $\begin{array}{c}1.595(0.741 \\
3.435)\end{array}$ & 0.233 \\
\hline & Yes & 150 & $131(87.3)$ & $19(12.7)$ & 1 & & 1 & \\
\hline Toilet & Absent & 206 & $163(79.1)$ & $43(20.9)$ & $2.412(1.031,5.640)$ & $0.042^{*}$ & $\begin{array}{c}0.513(0.040 \\
6.549)\end{array}$ & 0.608 \\
\hline & Present & 71 & $64(90.1)$ & $7(9.9)$ & 1 & & 1 & \\
\hline & Private & 31 & $29(93.5)$ & $2(6.5)$ & 1 & & 1 & \\
\hline Type of toilet & Open field & 209 & $165(78.9)$ & $44(21.1)$ & $\begin{array}{c}3.867(0.888 \\
16.834)\end{array}$ & 0.085 & $\begin{array}{c}4.795(0.212 \\
108.62)\end{array}$ & 0.613 \\
\hline & Public & 37 & $33(89.2)$ & $4(10.8)$ & $\begin{array}{c}1.758(0.300 \\
10.310)\end{array}$ & & $\begin{array}{c}1.675(0.223 \\
12.550)\end{array}$ & \\
\hline & No & 166 & $144(86.7)$ & $22(13.3)$ & 1 & & 1 & \\
\hline Eating raw meat & Yes & 111 & $83(74.8)$ & $28(25.2)$ & $2.208(1.187,4.106)$ & $0.012^{*}$ & $\begin{array}{c}2.477(1.252 \\
4.902)\end{array}$ & $0.009^{*}$ \\
\hline Eating raw & No & 150 & $119(79.3)$ & $31(20.7)$ & 1 & & 1 & \\
\hline vegetables & Yes & 127 & $108(85)$ & $19(15)$ & $0.675(0.361,1.265)$ & 0.220 & $\begin{array}{c}0.708(0.341 \\
1.471)\end{array}$ & 0.355 \\
\hline & Good & 92 & $81(88)$ & $11(12)$ & 1 & & 1 & \\
\hline Personal hygiene & Poor & 185 & $146(78.9)$ & $39(21.1)$ & $1.967(0.955,4.050)$ & 0.066 & $\begin{array}{c}1.295(0.499 \\
3.358)\end{array}$ & 0.595 \\
\hline
\end{tabular}

* = statically significant at $P<0.05$. 
personal and community hygiene such as frequent handwashing, fingernail cleaning, regular bathing, and washing of nightclothes and bed lining [45].

High family size, unsafe and inadequate provision of water, unhygienic living conditions, absence or improper utilization of latrine, not washing hands after toilet, and the habit of walking barefoot are significantly associated with IPIs $[7,14,22,49]$. But these factors did not found to be statistically associated with IPIs in the present study.

In the present study, we found IPIs statistically associated with eating raw meat with the odds of $1.66(\mathrm{AOR}=1.66$; $95 \%$ CI: 1.03, 2.67, $P=0.036$ ). This finding is congruent with the study finding around Lake Ziwai, Ethiopia [50].

One of the risk factors for G. lamblia was eating raw vegetables with the odds of 2.72 ( $\mathrm{AOR}=2.72 ; 95 \% \mathrm{CI}: 1.27$, $5.85 ; P=0.010 P=0.010)$. It is consistent with the study finding in East Wollega, Ethiopia [26]. The other associated risk factor was poor personal hygiene. Mothers with poor personal hygiene were 4.02 times ( $\mathrm{AOR}=4.015 ; 95 \% \mathrm{CI}$ : $1.46,11.07 ; P=0.007)$ more infected than their counterparts. This report is in agreement with the findings from Jawi Town, Northwest Ethiopia [51], and Goiânia, Goiás State, Brazil [36].

Taenia species was significantly associated with eating raw meat. Mothers who ate raw meat were 2.26 times more infected than their counterparts ( $\mathrm{AOR}=2.26$; $95 \% \mathrm{CI}: 1.13$, $4.55, P=0.02$ ). This finding was supported by the finding from around Lake Ziwai, Ethiopia [50]. However, other identified IPIs were not significantly associated with any of the potential risk factors.

\section{Conclusions}

This study indicated that there was a high prevalence of IPIs among pregnant women in the selected area. The most common detected intestinal parasites were Taenia species followed by G. lamblia, E. histolytica/dispar, and hookworms. Eating raw meat was an associated risk factor for IPIs. Eating raw vegetables and poor personal hygiene were predictors of G. lamblia, and eating raw meat was an associated risk factor for Taenia species. So, avoiding eating raw meat and vegetables, making and strengthening sanitation and hygiene programs, creating awareness about IPIs, making closed toilets, and routine deworming of mothers before pregnancy and on second and third trimesters are recommended.

5.1. Limitation. Use of single-season data, use of only wet mount and formol-ether concentration techniques to identify IPIs, and taking stool samples only in the daytime which may affect $E$. vermicularis load were the limitations of this study.

\section{Data Availability}

All the data generated or analyzed during this study are included in this published article (and its supplementary information files).

\section{Conflicts of Interest}

The authors have declared that no conflicts of interest exist.

\section{Acknowledgments}

The authors acknowledge Bahir Dar University for giving ethical clearance, Libokemkem Health Office for giving permeations to conduct this research, Yifag Health Center for allowing laboratory facilities, and the participants who voluntarily involved in this study.

\section{Supplementary Materials}

S1_File: ethical clearance letter. (Supplementary Materials)

\section{References}

[1] M. Norhayati, M. S. Fatmah, S. Yusof, and A. B. Edariah, "Intestinal parasitic infections in man: a review," Medical Journal of Malaysia, vol. 58, no. 2, pp. 296-306, 2003.

[2] L. Sapkota and M. Maharjan, "Anaemia association with intestinal parasitic infection in pregnant women attending antenatal clinic at Tribhuvan University teaching hospital," Journal of Advanced College of Engineering and Management, vol. 3, pp. 41-47, 2017.

[3] D. Gebretsadik, Y. Metaferia, A. Seid, G. M. Fenta, and A. Gedefie, "Prevalence of intestinal parasitic infection among children under 5 years of age at Dessie Referral Hospital: cross sectional study," BMC Research Notes, vol. 11, no. 1, p. 771, 2018.

[4] World Health Organization, "Soil-transmitted helminth infections," 2020, https://www.who.int/en/news-room/factsheets/detail/soil-transmitted-helminth-infections.

[5] A. Taghipour, S. Ghodsian, M. Jabbari, M. Olfatifar, A. Abdoli, and F. Ghaffarifar, "Global prevalence of intestinal parasitic infections and associated risk factors in pregnant women: a systematic review and meta-analysis," Transactions of the Royal Society of Tropical Medicine and Hygiene, vol. 115, no. 5, pp. 457-470, 2021.

[6] A. Derso, E. Nibret, and A. Munshea, "Prevalence of intestinal parasitic infections and associated risk factors among pregnant women attending antenatal care center at Felege Hiwot Referral Hospital, Northwest Ethiopia," BMC Infectious Diseases, vol. 16, no. 1, p. 530, 2016.

[7] D. A. Yesuf, L. T. Abdissa, E. A. Gerbi, and E. K. Tola, "Prevalence of intestinal parasitic infection and associated factors among pregnant women attending antenatal care at public health facilities in Lalo Kile district, Oromia, Western Ethiopia," BMC Research Notes, vol. 12, no. 1, p. 735, 2019.

[8] T. Hailu, B. Abera, W. Mulu, S. Kassa, A. Genanew, and A. Amor, "Prevalence and factors associated with intestinal parasitic infections among pregnant women in West Gojjam zone, northwest Ethiopia," Journal of Parasitology Research, vol. 2020, Article ID 8855362, 2020.

[9] A. Bolka and S. Gebremedhin, "Prevalence of intestinal parasitic infection and its association with anemia among pregnant women in Wondo Genet district, Southern Ethiopia: a cross-sectional study," BMC Infectious Diseases, vol. 19, no. 1, p. 483, 2019.

[10] S. Abhay, L. G. Simon, J. P. Hotez, and M. Tsuji, Medical Parasitology, pp. 195-221, Vademecum Parasitology Landes Bioscience, Austin, TX, USA, 2009. 
[11] Federal Democratic Republic of Ethiopia Population Census Commission, Summary and Statistical Report of the 2007 Population and Housing Census, Addis Ababa: Federal Democratic Republic of Ethiopia, Population Census Commission, Addis Ababa, Ethiopia, 2008.

[12] M. B. Shiferaw, A. M. Zegeye, and A. D. Mengistu, "Helminth infections and practice of prevention and control measures among pregnant women attending antenatal care at Anbesame Health Center, Northwest Ethiopia," BMC Research Notes, vol. 10, no. 1, pp. 274-275, 2017.

[13] F. Cox, Basic Laboratory Methods in Medical Parasitology, 1991. Sw. fr. 21.00 (Sw. fr. 14.70 in Developing Countries) $\$ 18.00$ (vii+ 114 pages) ISBN 924 1544104, World Health Organization, Geneva, Switzerland, 1992.

[14] A. Tigabu, S. Taye, M. Aynalem, and K. Adane, "Prevalence and associated factors of intestinal parasitic infections among patients attending Shahura Health Center, Northwest Ethiopia," BMC Research Notes, vol. 12, no. 1, p. 333, 2019.

[15] D. W. Hosmer Jr., S. Lemeshow, and R. X. Sturdivant, Applied Logistic Regression, John Wiley \& Sons, Hoboken, NJ, USA, 2013.

[16] M. G. Gebrehiwet, A. A. Medhaniye, and H. B. Alema, "Prevalence and associated factors of soil transmitted helminthes among pregnant women attending antenatal care in Maytsebri Primary Hospital, North Ethiopia," BMC Research Notes, vol. 12, no. 1, p. 644, 2019.

[17] E. Amuta, R. Houmsou, and S. Mker, "Knowledge and risk factors of intestinal parasitic infections among women in Makurdi, Benue State," Asian Pacific Journal of Tropical Medicine, vol. 3, no. 12, pp. 993-996, 2010.

[18] M. Getachew, D. Yewhalaw, K. Tafess, Y. Getachew, and A. Zeynudin, "Anaemia and associated risk factors among pregnant women in Gilgel Gibe dam area, Southwest Ethiopia," Parasites \& Vectors, vol. 5, no. 1, p. 296, 2012.

[19] Z. Gizaw, T. Adane, J. Azanaw, A. Addisu, and D. Haile, "Childhood intestinal parasitic infection and sanitation predictors in rural Dembiya, Northwest Ethiopia," Environmental Health and Preventive Medicine, vol. 23, no. 1, p. 26, 2018.

[20] A. F. Espinosa Aranzales, K. Radon, G. Froeschl, Á. M. Pinzón Rondón, and M. Delius, "Prevalence and risk factors for intestinal parasitic infections in pregnant women residing in three districts of Bogotá, Colombia," BMC Public Health, vol. 18, no. 1, p. 1071, 2018.

[21] A. Wekesa, C. Mulambalah, C. Muleke, and R. Odhiambo, "Intestinal helminth infections in pregnant women attending antenatal clinic at Kitale District Hospital, Kenya," Journal of parasitology research, vol. 2014, Article ID 823923, 2014.

[22] B. E. Feleke and T. H. Jember, "Prevalence of helminthic infections and determinant factors among pregnant women in Mecha district, Northwest Ethiopia: a cross sectional study," BMC Infectious Diseases, vol. 18, no. 1, p. 373, 2018.

[23] S. Phuanukoonnon, A. Michael, W. S. Kirarock, W. S. Pomat, and A. H. van den Biggelaar, "Intestinal parasitic infections and anaemia among pregnant women in the highlands of Papua New Guinea," Papua New Guinea Medical Journal, vol. 56, no. 3/4, pp. 119-125, 2013.

[24] A. J. Rodríguez-Morales, R. A. Barbella, C. Case et al., "Intestinal parasitic infections among pregnant women in Venezuela," Infectious Diseases in Obstetrics and Gynecology, vol. 2006, Article ID 23125, 2006.

[25] A. Bekele, M. Tilahun, and A. Mekuria, "Prevalence of anemia and its associated factors among pregnant women attending antenatal care in health institutions of Arba Minch town,
Gamo Gofa Zone, Ethiopia: a cross-sectional study,” Anemia, vol. 2016, Article ID 1073192, 2016.

[26] H. M. Mengist, O. Zewdie, and A. Belew, "Intestinal helminthic infection and anemia among pregnant women attending ante-natal care (ANC) in East Wollega, Oromia, Ethiopia," BMC Research Notes, vol. 10, no. 1, p. 440, 2017.

[27] J. Saki, S. Khademvatan, M. Foroutan-Rad, and M. Gharibzadeh, "Prevalence of intestinal parasitic infections in Haftkel County, Southwest of Iran," International Journal of Infection, vol. 4, no. 4, Article ID e15593, 2017.

[28] World Health Organization, "Taeniasis/cysticercosis key facts," 2020, https://www.who.int/news-room/fact-sheets/ detail/taeniasis-cysticercosis\#: :text=Key\%20facts,Taenia\%20 saginata\%20and\%20Taenia\%20asiatica.\&text=solium\%20tae niasis\%20is\%20acquired\%20by.in\%20undercooked\%20and\% 20infected\%20pork.

[29] F. Weldekidan, M. Kote, M. Girma, N. Boti, and T. Gultie, "Determinants of anemia among pregnant women attending antenatal clinic in public health facilities at Durame Town: unmatched case control study," Anemia, vol. 2018, Article ID 8938307, 2018.

[30] T. Hailu, S. Kassa, B. Abera, W. Mulu, and A. Genanew, "Determinant factors of anaemia among pregnant women attending antenatal care clinic in Northwest Ethiopia," Tropical Diseases, Travel Medicine and Vaccines, vol. 5, no. 1, p. 13, 2019.

[31] L. Gedefaw, A. Ayele, Y. Asres, and A. Mossie, "Anaemia and associated factors among pregnant women attending antenatal care clinic in Walayita Sodo town, Southern Ethiopia," Ethiopian Journal of health sciences, vol. 25, no. 2, pp. 155-164, 2015.

[32] W. Espelage, M. an der Heiden, K. Stark, and K. Alpers, "Characteristics and risk factors for symptomatic Giardia lamblia infections in Germany," BMC Public Health, vol. 10, no. 1, p. 41, 2010.

[33] T. Wegayehu, T. Tsalla, B. Seifu, and T. Teklu, "Prevalence of intestinal parasitic infections among highland and lowland dwellers in Gamo area, South Ethiopia," BMC Public Health, vol. 13, no. 1, p. 151, 2013.

[34] A. Jejaw, E. Zemene, Y. Alemu, and Z. Mengistie, "High prevalence of Schistosoma mansoni and other intestinal parasites among elementary school children in Southwest Ethiopia: a cross-sectional study," BMC Public Health, vol. 15, no. 1, pp. 600-607, 2015.

[35] A. M. Mahande and M. J. Mahande, "Prevalence of parasitic infections and associations with pregnancy complications and outcomes in northern Tanzania: a registry-based cross-sectional study," BMC Infectious Diseases, vol. 16, no. 1, p. 78, 2016.

[36] M. D. G. C. Pereira, E. R. Atwill, and A. P. Barbosa, "Prevalence and associated risk factors for Giardia lamblia infection among children hospitalized for diarrhea in Goiânia, Goiás State, Brazil," Revista do Instituto de Medicina Tropical de São Paulo, vol. 49, no. 3, pp. 139-145, 2007.

[37] G. F. Chami, A. Fenwick, E. Bulte et al., "Influence of Schistosoma mansoni and hookworm infection intensities on anaemia in Ugandan villages," PLoS Neglected Tropical Diseases, vol. 9, no. 10, Article ID e0004193, 2015.

[38] N. Ivoke, N. Ikpor, O. Ivoke et al., "Geophagy as risk behaviour for gastrointestinal nematode infections among pregnant women attending antenatal clinics in a humid tropical zone of Nigeria," African Health Sciences, vol. 17, no. 1, pp. 24-31, 2017. 
[39] S. Baidoo, S. Tay, K. Obiri-Danso, and H. Abruquah, "Intestinal helminth infection and anaemia during pregnancy: a community-based study in Ghana," African Journal of Microbiology Research, vol. 2, no. 16, pp. 9-13, 2010.

[40] D. Yang, Y. Yang, Y. Wang et al., "Prevalence and risk factors of Ascaris lumbricoides, Trichuris trichiura and Cryptosporidium infections in elementary school children in Southwestern China: a school-based cross-sectional study," International Journal of Environmental Research and Public Health, vol. 15, no. 9, p. 1809, 2018.

[41] N. Abebe, B. Erko, G. Medhin, and N. Berhe, "Clinico-epidemiological study of schistosomiasis mansoni in wajatimuga, district of Alamata, Northern Ethiopia," Parasites \& Vectors, vol. 7, no. 1, p. 158, 2014.

[42] C. Tonga, C. Ngo Bayoi, F. C. Tchanga et al., "Schistosomiasis among pregnant women in Njombe-Penja Health district, Cameroon," The Journal of Infection in Developing Countries, vol. 13, no. 12, pp. 1150-1158, 2019.

[43] M. Alem, B. Enawgaw, A. Gelaw, T. Kenaw, M. Seid, and Y. Olkeba, "Prevalence of anemia and associated risk factors among pregnant women attending antenatal care in Azezo Health Center Gondar Town, Northwest Ethiopia," Journal of Interdisciplinary Histopathology, vol. 1, no. 3, pp. 137-144, 2013.

[44] H. T. AL-Marsome, "Prevalence of hymenolepis nana infections in Abu-Ghraib City/Baghdad/Iraq," Iraqi Academic Scientific Journal, vol. 11, no. 4, pp. 581-584, 2012.

[45] C. J. Paniker and S. Ghosh, Paniker's Textbook of Medical Parasitology, JP Medical Ltd., New Delhi, India, 2017.

[46] Y. Terefe, K. Ross, and H. Whiley, "Strongyloidiasis in Ethiopia: systematic review on risk factors, diagnosis, prevalence and clinical outcomes," Infectious diseases of poverty, vol. 8, no. 1, p. 53, 2019.

[47] J. Alli, I. Okonko, A. Kolade et al., "Prevalence of intestinal nematode infection among pregnant women attending antenatal clinic at the University College Hospital, Ibadan, Nigeria," Advances in Applied Science Research, vol. 2, no. 4, pp. 1-13, 2011.

[48] A. K. A.-H. Ahmed and A.-M. A. H. Al-Rubaea, "Prevalence of parasitic infection and relationship with anemia in AlDoullab village, Babylon province, Iraq," The Egyptian Journal of Experimental Biology (Zoology), vol. 9, no. 2, pp. 231-236, 2013.

[49] D. J. Tesfaye, "Prevalence of intestinal helminthiases and associated factors among pregnant women attending antenatal clinic of Nigist Eleni Mohammed Memorial Hospital, Hossana, Southern Ethiopia," Open Access Library Journal, vol. 2, no. 7, p. 1, 2015.

[50] A. Sisay and B. Lemma, "Assessment on the prevalence and risk factors of gastrointestinal parasites on schoolchildren at Bochesa Elementary School, around Lake Zwai, Ethiopia," BMC Research Notes, vol. 12, no. 1, p. 410, 2019.

[51] B. Sitotaw, H. Mekuriaw, and D. Damtie, "Prevalence of intestinal parasitic infections and associated risk factors among Jawi primary school children, Jawi town, North-West Ethiopia," BMC Infectious Diseases, vol. 19, no. 1, p. 341, 2019. 